\title{
A Selection Method Based on MAGDM with Interval-Valued Intuitionistic Fuzzy Sets
}

\author{
Gai-Li Xu, ${ }^{1,2}$ Shu-Ping Wan, ${ }^{2}$ and Xiao-Lan Xie ${ }^{3}$ \\ ${ }^{1}$ College of Science, Guilin University of Technology, Guilin 541004, China \\ ${ }^{2}$ College of Information Technology, Jiangxi University of Finance and Economics, Nanchang 330013, China \\ ${ }^{3}$ College of Information Science and Engineering, Guilin University of Technology, Guilin 541002, China
}

Correspondence should be addressed to Gai-Li Xu; jiali0706@126.com

Received 3 December 2014; Accepted 11 May 2015

Academic Editor: Julien Bruchon

Copyright (C) 2015 Gai-Li Xu et al. This is an open access article distributed under the Creative Commons Attribution License, which permits unrestricted use, distribution, and reproduction in any medium, provided the original work is properly cited.

\begin{abstract}
As the cloud computing develops rapidly, more and more cloud services appear. Many enterprises tend to utilize cloud service to achieve better flexibility and react faster to market demands. In the cloud service selection, several experts may be invited and many attributes (indicators or goals) should be considered. Therefore, the cloud service selection can be regarded as a kind of Multiattribute Group Decision Making (MAGDM) problems. This paper develops a new method for solving such MAGDM problems. In this method, the ratings of the alternatives on attributes in individual decision matrices given by each expert are in the form of interval-valued intuitionistic fuzzy sets (IVIFSs) which can flexibly describe the preferences of experts on qualitative attributes. First, the weights of experts on each attribute are determined by extending the classical gray relational analysis (GRA) into IVIF environment. Then, based on the collective decision matrix obtained by aggregating the individual matrices, the score (profit) matrix, accuracy matrix, and uncertainty (risk) matrix are derived. A multiobjective programming model is constructed to determine the attribute weights. Subsequently, the alternatives are ranked by employing the overall scores and uncertainties of alternatives. Finally, a cloud service selection problem is provided to illustrate the feasibility and effectiveness of the proposed methods.
\end{abstract}

\section{Introduction}

Cloud computing [1-4] is the latest computing paradigm that delivers hardware and software resources as virtualization services in which users are free from the burden of worrying about the low-level system administration details. In recent years, cloud computing is developing rapidly and has provided enterprises with many advantages such as flexibility, business agility, and pay-as-you-go cost structure. As a result, many enterprises with limited financial and human resources are increasingly adopting cloud computing to deliver their business services and products online to extend their business markets. In many domains, multiple cloud services often supply similar functional properties. For example, in Customer Relationship Management (CRM), CRM venders offer functionally equivalent cloud services, such as Microsoft Dynamic CRM, Salesforce Sales Cloud, SAP Sales on Demand, and Oracle Cloud CRM. However, for enterprises, which lack cloud computing knowledge, it is difficult to select an appropriate candidate from a set of functionally equivalent cloud services. Therefore, it is necessary for enterprises to invite several related experts to evaluate the potential candidates from several indicators (attributes), such as payment, performance, reputation, scalability, and security. The selection of cloud services has attracted attention and many methods have been presented to guide enterprises in selecting the cloud services. Roughly, these methods may be divided into two categories and briefly reviewed as follows, respectively.

The first category is the Multiattribute Decision Making (MADM) methods. According to the key performance indicators defined by Siegel and Perdue [5], Garg et al. [6] proposed the cloud service ranking framework using the Analytic Hierarchy Process (AHP) technique. Menzel et al. [7] utilized the Analytic Network Process (ANP) to develop a Multicriteria Comparison Method which is used to select 
Infrastructure-as-a-Service (IaaS). Limam and Boutaba [8] presented a trustworthiness-based service selection method based on the Multiple Attribute Utility Theory (MAUT). By employing the Elimination and Choice Expressing Reality (ELECTRE) method, Silas et al. [9] developed a cloud service selection middleware to help cloud users select desired cloud service. Saripalli and Pingali [10] discussed Simple Additive Weighting (SAW) methods to rank alternatives in a decision problem of cloud service adoption. Zhao et al. [11] suggested a SAW-based service searching and scheduling algorithm to obtain a set of ranked services.

The second category is the optimization approaches. Chang et al. [12] designed a dynamic programming algorithm by maximizing the overall survival probability to select cloud storage providers. Sundareswaran et al. [13] selected cloud service with a greedy algorithm method which can make experts retrieve information fast. In order to help service providers to select Software-as-a-Service (SaaS) services with multitenants, He et al. [14] explored three types of optimization algorithm, including integer programming, skyline, and greedy algorithm, and proposed a quality of service- (QoS-) driven optimization framework. By minimizing costs and risks, Martens et al. [15] constructed a scalable mathematical decision model to select cloud service. Yang et al. [16] built a Markov decision process model to guarantee the nearoptimal performance in a changing environment by dynamically adjusting the components of a service composition.

The aforementioned methods seem to be effective and applicable for selecting cloud services. However, they have the following shortcomings.

(1) The decision making in methods [13-15] is single Multiattribute Decision Making (MADM); that is, only one expert participates in the decision making and gives assessment information of alternatives with respect to several attributes. Since every expert is good at only some fields rather than all fields, the reliability of some information given by the expert is a little doubtful.

(2) Current methods $[9,12]$ are more focused on quantitative attributes measured via precise numerical values, such as response time, storage space, and latency time. Nevertheless, in cloud service, some qualitative attributes (such as reputation and security) usually play important roles, but they do not gain enough attention.

(3) In existing methods $[12,13,16]$, the assessment values (attribute values) are crisp numbers, which is somewhat unrealistic. Due to the inherent vagueness of human preferences as well as the fuzziness and uncertainty of objects, it is more suitable to express the assessment values as fuzzy numbers [17-20].

(4) The attribute weights provided by experts are given a priori in methods $[8,10,11]$, which always cannot avoid subjective randomness of the expert's preference. Furthermore, with increasing complexity in many real decision situations, it is difficult for expert to provide precise and complete preference information due to time pressure and lack of data.

One of the reasons leading to the above shortcomings is that the fuzziness and uncertainty are not fully considered during the decision making process. The fuzzy set (FS) theory introduced by Zadeh [21] is a very useful tool to describe fuzzy and uncertain information. Based on the FS theory, Atanassov [22] presented the intuitionistic fuzzy set (IFS), which considers the membership (satisfaction) degree, nonmembership (dissatisfaction) degree, and hesitant degree simultaneously. Subsequently, Atanassov and Gargov [23] generalized IFS and presented interval-valued intuitionistic fuzzy set (IVIFS) that describes the membership and nonmembership degrees as intervals. Compared with the FS and IFS, IVIFS is more suitable to express the fuzziness and uncertainty and has been widely used in many fields [24-28].

According to IVIFS theory, to overcome the aforementioned shortcomings, we investigate the cloud service selection problems with IVIFSs and develop a novel method. The proposed method has the following key characteristics.

(1) The selection of cloud service is regarded as a Multiattribute Group Decision Making (MAGDM) problem that several experts are invited to evaluate the potential cloud services, whereas it is considered as a single MADM problem in methods [6-11]. With increasing complexity and the limit knowledge owned by single expert, in order to increase the quality of cloud service, it is more reasonable and reliable for enterprises to invite multiple experts to participate in making decision.

(2) The assessment values given by experts are expressed as IVIFSs. Compared with the crisp number, IVIFS is more flexible to measure the qualitative attributes since IVIFS considers membership, nonmembership, and hesitant degrees which are expressed as intervals. Additionally, it is easier for experts to supply assessment values with IVIFSs in the increasing uncertain and complex environment.

(3) By extending the classical gray relational analysis (GRA) [29] into IVIF environment, a new approach is proposed to determine the weights of experts. A notable characteristic of the proposed approach is that the obtained weights of each expert are different with respect to different attributes.

(4) For MAGDM problems with incomplete information on attributes, a multiobjective programming model is constructed to objectively determine the attribute weights, which can avoid the subjective randomness appearing in the methods $[8,10,11]$. Moreover, it is easier for experts to give partial information on attribute weights than to assign a crisp number to the attribute weights.

The rest of this paper unfolds as follows. Some preliminaries about IVIFSs and the classical GRA method are 
introduced in Section 2. In Section 3, a new method is proposed to solve MAGDM problems with IVIFSs and incomplete attribute weight information. In addition, a framework of decision supporting system (DSS) is constructed. In Section 4, a cloud service selection example is provided to illustrate the applicability of the proposed method and comparison analysis is conducted. Finally, the conclusions are discussed in Section 5.

\section{Preliminaries}

In this section, we introduce some basic concepts related to interval-valued fuzzy set (IVIFS) and gray relational analysis (GRA).

\subsection{Interval-Valued Intuitionistic Fuzzy Set}

Definition 1 (see [23]). Let $X=\left\{x_{1}, x_{2}, \ldots, x_{n}\right\}$ be a nonempty set of the universe. An IVIFS $\widetilde{A}$ in $X$ is defined as

$$
\begin{aligned}
\widetilde{A} & =\left\{\left(x_{i},\left[\mu_{\widetilde{A}}^{L}\left(x_{i}\right), \mu_{\widetilde{A}}^{R}\left(x_{i}\right)\right],\left[v_{\widetilde{A}}^{L}\left(x_{i}\right), v_{\widetilde{A}}^{R}\left(x_{i}\right)\right]\right) \mid x_{i}\right. \\
& \in X\},
\end{aligned}
$$

where $\left[\mu_{\widetilde{A}}^{L}\left(x_{i}\right), \mu_{\widetilde{A}}^{R}\left(x_{i}\right)\right]$ and $\left[v_{\widetilde{A}}^{L}\left(x_{i}\right), v_{\widetilde{A}}^{R}\left(x_{i}\right)\right]$ denote the intervals of membership degree and nonmembership degree of element $x_{i} \in \widetilde{A}$, respectively, satisfying $\mu_{\widetilde{A}}^{R}\left(x_{i}\right)+v_{\widetilde{A}}^{R}\left(x_{i}\right) \leq 1$, $0 \leq \mu_{\widetilde{A}}^{L}\left(x_{i}\right) \leq \mu_{\widetilde{A}}^{R}\left(x_{i}\right) \leq 1$, and $0 \leq v_{\widetilde{A}}^{L}\left(x_{i}\right) \leq v_{\widetilde{A}}^{R}\left(x_{i}\right) \leq 1$ for all $x_{i} \in X . \pi_{\widetilde{A}}\left(x_{i}\right)=\left[1-\mu_{\widetilde{A}}^{R}\left(x_{i}\right)-v_{\widetilde{A}}^{R}\left(x_{i}\right), 1-\mu_{\widetilde{A}}^{L}\left(x_{i}\right)-v_{\widetilde{A}}^{L}\left(x_{i}\right)\right]$ is called the interval-valued intuitionistic hesitant degree of IVIFS $\widetilde{A}$. For any $x_{i} \in X$, if $\mu_{\widetilde{A}}^{L}\left(x_{i}\right)=\mu_{\widetilde{A}}^{R}\left(x_{i}\right)$ and $v_{\widetilde{A}}^{L}\left(x_{i}\right)=$ $v_{\widetilde{A}}^{R}\left(x_{i}\right)$, then $\widetilde{A}$ is reduced to an IFS.

$\mathrm{Xu}$ [30] called the pair $\tilde{\alpha}=\left(\mu_{\tilde{\alpha}}\left(x_{i}\right), v_{\tilde{\alpha}}\left(x_{i}\right)\right)$ an intervalvalued intuitionistic fuzzy number (IVIFN) and denoted an IVIFN by $\tilde{\alpha}=([a, b],[c, d])$, where $[a, b] \subseteq[0,1],[c, d] \subseteq$ $[0,1], b+d \leq 1$.

Definition 2 (see [30]). Let $\tilde{\alpha}_{1}=\left(\left[a_{1}, b_{1}\right],\left[c_{1}, d_{1}\right]\right), \tilde{\alpha}_{2}=$ $\left(\left[a_{2}, b_{2}\right],\left[c_{2}, d_{2}\right]\right)$, and $\tilde{\alpha}=([a, b],[c, d])$ be three IVIFNs; then

(1) $(\widetilde{\alpha})^{c}=([c, d],[a, b])$;

(2) $\tilde{\alpha}_{1}+\widetilde{\alpha}_{2}=\left(\left[a_{1}+a_{2}-a_{1} a_{2}, b_{1}+b_{2}-b_{1} b_{2}\right],\left[c_{1} c_{2}, d_{1} d_{2}\right]\right)$;

(3) $\lambda \widetilde{\alpha}=\left(\left[1-(1-a)^{\lambda}, 1-(1-b)^{\lambda}\right],\left[c^{\lambda}, d^{\lambda}\right]\right), \lambda>0$.

Definition 3 (see [30]). Let $\tilde{\alpha}_{j}=\left(\left[a_{j}, b_{j}\right],\left[c_{j}, d_{j}\right]\right)(j=$ $1,2, \ldots, n)$ be a collection of IVIFNs. If

$$
\operatorname{IVIFWA}_{\omega}\left(\widetilde{\alpha}_{1}, \widetilde{\alpha}_{2}, \ldots, \widetilde{\alpha}_{n}\right)=\sum_{j=1}^{n} \omega_{j} \widetilde{\alpha}_{j},
$$

then the IVIFWA is called an interval-valued intuitionistic fuzzy weighted averaging (IVIFWA) operator of dimension $n$, where $\boldsymbol{\omega}=\left(\omega_{1}, \omega_{2}, \ldots, \omega_{n}\right)^{T}$ is a weight vector of $\widetilde{\alpha}_{j}$ with $\omega_{j} \in[0,1]$ and $\sum_{j=1}^{n} \omega_{j}=1$.
The aggregated value determined by the IVFWA operator is also an IVIFN; that is,

$$
\begin{aligned}
& \operatorname{IVIFWA}_{\omega}\left(\widetilde{\alpha}_{1}, \widetilde{\alpha}_{2}, \ldots, \widetilde{\alpha}_{n}\right) \\
& =\left(\left[1-\prod_{j=1}^{n}\left(1-a_{j}\right)^{\omega_{j}}, 1-\prod_{j=1}^{n}\left(1-b_{j}\right)^{\omega_{j}}\right],\right. \\
& \left.\left[\prod_{j=1}^{n} c_{j}^{\omega_{j}}, \prod_{j=1}^{n} d_{j}^{\omega_{j}}\right]\right) .
\end{aligned}
$$

Definition 4 (see $[30])$. Let $\tilde{\alpha}=([a, b],[c, d])$ be an IVIFN. Then

$$
\begin{aligned}
& s(\widetilde{\alpha})=\frac{1}{2}(a+b-c-d), \\
& h(\widetilde{\alpha})=\frac{1}{2}(a+c+b+d)
\end{aligned}
$$

are, respectively, called the score function and accuracy function of the IVIFN $\widetilde{\alpha}$, where $s(\widetilde{\alpha}) \in[-1,1]$ and $h(\widetilde{\alpha}) \epsilon$ $[0,1]$ can be considered as net membership and accuracy degree, respectively.

Since $s(\tilde{\alpha}) \in[-1,1]$, when many score functions are aggregated with linear weighted summation method, it maybe appears that positive score functions are offset by negative score functions. Therefore, we normalize the score function and make it belong to $[0,1]$.

Given a variable $y \in[-1,1]$, if we define

$$
f(y)=\frac{y+1}{2},
$$

then $f(y)$ cannot only retain the monotonicity of the variable $y$ but also map $y$ for $[0,1]$. Hence, we modify the score function in Definition 4 and define a new score function of IVIFN $\tilde{\alpha}$.

Definition 5. Let $\widetilde{\alpha}=([a, b],[c, d])$ be an IVIFN. Then

$$
s^{*}(\widetilde{\alpha})=\frac{1}{2}(s(\widetilde{\alpha})+1)
$$

is called a normalized score function, where $s(\widetilde{\alpha})=(1 / 2)(a-$ $c+b-d)$. Obviously, $s^{*}(\widetilde{\alpha}) \in[0,1]$.

Definition 6. Let $\widetilde{\alpha}=([a, b],[c, d])$ be an IVIFN. Then

$$
\gamma(\widetilde{\alpha})=1-h(\widetilde{\alpha})
$$

is called an uncertainty function, where $h(\widetilde{\alpha})=(1 / 2)(a+c+$ $b+d)$.

Let $\widetilde{\alpha}=([a, b],[c, d])$ be an assessment value of the cloud service $x$ with respect to the attribute (indicator) $\tilde{\alpha}$. Then the normalized score function $s^{*}(\widetilde{\alpha})$ and the uncertainty function $\gamma(\widetilde{\alpha})$ can be, respectively, interpreted as the "net profit" and "risk" provided by cloud service $x$ on attribute $\widetilde{\alpha}$. Hence, the bigger the $s^{*}(\widetilde{\alpha})$ and the smaller the $\gamma(\widetilde{\alpha})$, the better the cloud service $x$. In the following, an order relationship between IVIFNs is given. 
Definition 7. Let $\tilde{\alpha}_{1}=\left(\left[a_{1}, b_{1}\right],\left[c_{1}, d_{1}\right]\right), \tilde{\alpha}_{2}=\left(\left[a_{2}, b_{2}\right],\left[c_{2}\right.\right.$, $\left.d_{2}\right]$ ) be two IVIFNs; then

(1) If $s^{*}\left(\widetilde{\alpha}_{1}\right)<s^{*}\left(\widetilde{\alpha}_{2}\right)$, then $\widetilde{\alpha}_{1}<\widetilde{\alpha}_{2}$.

(2) If $s^{*}\left(\widetilde{\alpha}_{1}\right)=s^{*}\left(\widetilde{\alpha}_{2}\right)$, then

(i) If $\gamma\left(\widetilde{\alpha}_{1}\right)=\gamma\left(\widetilde{\alpha}_{2}\right)$, then $\widetilde{\alpha}_{1}=\widetilde{\alpha}_{2}$.

(ii) If $\gamma\left(\widetilde{\alpha}_{1}\right)>\gamma\left(\widetilde{\alpha}_{2}\right)$, then $\widetilde{\alpha}_{1}<\widetilde{\alpha}_{2}$.

Definition 8. Let $\widetilde{\alpha}_{1}=\left(\left[a_{1}, b_{1}\right],\left[c_{1}, d_{1}\right]\right), \widetilde{\alpha}_{2}=\left(\left[a_{2}, b_{2}\right],\left[c_{2}\right.\right.$, $\left.d_{2}\right]$ ) be two IVIFNs; the Euclidean distance between $\widetilde{\alpha}_{1}$ and $\tilde{\alpha}_{2}$ is defined as follows:

$$
\begin{aligned}
& d\left(\widetilde{\alpha}_{1}, \widetilde{\alpha}_{2}\right) \\
& =\frac{1}{2} \sqrt{\left(a_{1}-a_{2}\right)^{2}+\left(b_{1}-b_{2}\right)^{2}+\left(c_{1}-c_{2}\right)^{2}+\left(d_{1}-d_{2}\right)^{2}} .
\end{aligned}
$$

2.2. Gray Relation Analysis. GRA Theorem is an important part of Gray Theorem developed by Deng [29]. GRA investigates uncertain relationship between one main factor and all other factors in a system and has been used in a wide variety of decision making environments, such as supplier selection [31], material selection [32], and water protection strategy evaluation [33]. follows

The details of the classical GRA method are presented as

(i) Calculate the normalized decision matrix.

Let $\mathbf{F}=\left(f_{i j}\right)_{m \times n}$ be a decision matrix. The normalized matrix $\mathbf{R}=\left(r_{i j}\right)_{m \times n}$ is calculated as

$$
r_{i j}= \begin{cases}\frac{\max _{i} f_{i j}-f_{i j}}{\max _{i} f_{i j}-\min _{i} f_{i j}} & i=1,2, \ldots, m ; j=1,2, \ldots, n ; j \in \text { cost attributes } \\ \frac{f_{i j}-\min _{i} f_{i j}}{\max _{i} f_{i j}-\min _{i} f_{i j}} & i=1,2, \ldots, m ; j=1,2, \ldots, n ; j \in \text { benefit attributes. }\end{cases}
$$

(ii) Generate comparability sequences $r_{i}=\left(r_{i 1}, r_{i 2}, \ldots\right.$, $\left.r_{i n}\right)(i=1,2, \ldots, m)$ and a reference sequence is $r_{0}=\left(r_{01}, r_{02}, \ldots, r_{0 n}\right)$. For example, we can take $r_{0 j}=$ $\max _{i}\left\{r_{i j}\right\}(j=1,2, \ldots, n)$.

(iii) Compute the gray relational coefficient between the comparability sequence $r_{i}$ and the reference sequence $r_{0}$ by the following formula:

$$
\begin{aligned}
\xi\left(r_{i j}, r_{0 j}\right)=\frac{d^{-}+\tau d^{+}}{d\left(r_{i j}, r_{0 j}\right)+\tau d^{+}} & \\
& (i=1,2, \ldots, m ; j=1,2, \ldots, n),
\end{aligned}
$$

where $d\left(r_{i j}, r_{0 j}\right)=\left|r_{i j}-r_{0 j}\right|, d^{-}=\min _{1 \leq i \leq m} \min _{1 \leq j \leq n} d\left(r_{i j}, r_{0 j}\right)$, $d^{+}=\max _{1 \leq i \leq m} \max _{1 \leq j \leq n} d\left(r_{i j}, r_{0 j}\right)$, and $\tau \in[0,1]$ is a distinguishing coefficient. Usually, $\tau=0.5$.

(iv) Calculate the gray relational grade between $r_{i}$ and $r_{0}$; that is,

$$
\xi_{i}=\sum_{j=1}^{n} \omega_{j} \xi_{i j},
$$

where $\boldsymbol{\omega}=\left(\omega_{1}, \omega_{2}, \ldots, \omega_{n}\right)^{T}$ is a weight vector satisfying $\omega_{j} \in[0,1]$ and $\sum_{j=1}^{n} \omega_{j}=1$. The bigger the $\xi_{i}$, the closer the sequence $r_{i}$ to the sequence $r_{0}$.

\section{A Novel Method for MAGDM with IVIFSs and Incomplete Attribute Weight Information}

In this section, a new method is proposed to handle MAGDM with IVIFSs. The proposed method includes determination of the weights of experts and identification of attribute weights.
Let $A=\left\{A_{1}, A_{2}, \ldots, A_{m}\right\}$ be the set of $m$ feasible alternatives, let $U=\left\{u_{1}, u_{2}, \ldots, u_{n}\right\}$ be the set of attributes, and let $E=\left\{e_{1}, e_{2}, \ldots, e_{t}\right\}$ be the set of decision makers (DMs). Assume that $\boldsymbol{\omega}=\left(\omega_{1}, \omega_{2}, \ldots, \omega_{n}\right)^{T}$ is an attribute weight vector, where $\omega_{j} \in[0,1]$ and $\sum_{j=1}^{n} \omega_{j}=1$. Let the individual decision matrix given by expert $e_{k}$ be $\widetilde{\mathbf{F}}^{k}=\left(\widetilde{f}_{i j}^{k}\right)_{m \times n}$, where $\widetilde{f}_{i j}^{k}=\left(\left[a_{i j}^{k}, b_{i j}^{k}\right],\left[c_{i j}^{k}, d_{i j}^{k}\right]\right)$ is an IVIFN for the alternative $A_{i}$ with respect to attribute $u_{j}$. In this paper, $\left[a_{i j}^{k}, b_{i j}^{k}\right]$ and $\left[c_{i j}^{k}, d_{i j}^{k}\right]$ provided by the expert $e_{k}$ are, respectively, the satisfaction (agreeing) degree interval and dissatisfaction (disagreeing) degree interval of the $i$ th cloud service $A_{i}$ with respect to the $j$ th attribute (indicator) $u_{j}$.

3.1. Determine the Weights of Experts by the Extended GRA Method. Due to the fact that each expert is skilled in some fields rather than all fields, it is more reasonable that the weights of each expert with respect to different attributes should be assigned different values. However, the weights of each expert obtained with the existing methods [34-37] are the same.

Let $\lambda_{j}^{k}$ be the weight of expert $e_{k}$ with respect to attribute $u_{j}$. Generally, for the attribute $u_{j}$, the closer the attribute values of all alternatives given by expert $e_{k}$ are to those given by all other $t-1$ experts, the more similar the information provided by the expert $e_{k}$ is to that implied by the group. Consequently, the weight of expert $e_{k}$ should be assigned a greater value. Bearing this idea in mind, we present a novel method to determine the weights of experts by extending classical GRA method.

Given the decision matrices $\widetilde{\mathbf{F}}^{k}=\left(\tilde{f}_{i j}^{k}\right)_{m \times n}(k=1,2, \ldots$, $t$ ), the elements $\tilde{f}_{i j}^{k}$ can be normalized as 


$$
\begin{aligned}
& \widetilde{r}_{i j}^{k} \\
& = \begin{cases}f_{i j}^{k} & i=1,2, \ldots, m ; j=1,2, \ldots, n ; j \in \text { benefit attributes } \\
\left(f_{i j}^{k}\right)^{c} & i=1,2, \ldots, m ; j=1,2, \ldots, n ; j \in \text { cost attributes. }\end{cases}
\end{aligned}
$$

The normalized decision matrices can be denoted by $\widetilde{\mathbf{R}}^{k}=$ $\left(\widetilde{r}_{i j}^{k}\right)_{m \times n}(k=1,2, \ldots, t)$.

Let $\widetilde{\mathbf{r}}_{j}^{k}=\left(\widetilde{r}_{1 j}^{k}, \widetilde{r}_{2 j}^{k}, \ldots, \widetilde{r}_{m j}^{k}\right)$ be the reference sequence and let all other sequences $\widetilde{\mathbf{r}}_{j}^{l}=\left(\vec{r}_{1 j}^{l}, \vec{r}_{2 j}^{l}, \ldots, \widetilde{r}_{m j}^{l}\right)(l=$ $1,2, \ldots, t, l \neq k$ ) be comparability sequences. Then, for the attribute $u_{j}$, the gray relational coefficient between $\widetilde{r}_{j}^{k}$ and $\vec{r}_{j}^{l}$ with respect to alternative $A_{i}$ is defined as

$$
\xi_{i j}^{l k}=\xi\left(\widetilde{r}_{i j}^{l}, \widetilde{r}_{i j}^{k}\right)=\frac{d_{j}^{k-}+\tau d_{j}^{k+}}{d\left(\widetilde{r}_{i j}^{l}, \tilde{r}_{i j}^{k}\right)+\tau d_{j}^{k+}},
$$

where $d\left(\widetilde{r}_{i j}^{l}, \widetilde{r}_{i j}^{k}\right)$ is the distance between $\widetilde{r}_{i j}^{l}$ and $\widetilde{r}_{i j}^{k}$ $(\operatorname{see}(9)), d_{j}^{k-}=\min _{1 \leq l \leq s, l \neq k} \min _{1 \leq i \leq m} d\left(\widetilde{r}_{i j}^{l}, \widetilde{r}_{i j}^{k}\right), d_{j}^{k+}=$ $\max _{1 \leq l \leq s, l \neq k} \max _{1 \leq i \leq m} d\left(\widetilde{r}_{i j}^{l}, \widetilde{r}_{i j}^{k}\right)$, and $\tau=0.5$.

Thus, the matrix of gray relational coefficient between $\vec{r}_{i j}$ and $\widetilde{r}_{i j}^{k}$ is constructed as

$$
\xi_{j}^{k}=\left(\xi_{i j}^{l k}\right)_{(t-1) \times m}
$$

where $l=1,2, \ldots, t, l \neq k$. as

The gray relational grade between $\widetilde{r}_{j}^{k}$ and $\vec{r}_{j}^{l}$ is calculated

$$
\eta\left(\vec{r}_{j}^{l}, \widehat{r}_{j}^{k}\right)=\frac{1}{m} \sum_{i=1}^{m} \xi_{i j}^{l k}
$$

The gray relational grade $\eta\left(\widetilde{r}_{j}^{k}, \vec{r}_{j}^{l}\right)$ describes the degree of closeness between sequence $\widetilde{r}_{j}^{k}$ and sequence $\widetilde{r}_{j}^{l}$. In other words, $\eta\left(\widetilde{r}_{j}^{k}, \widetilde{r}_{j}^{l}\right)$ indicates the similarity degree between the information given by $\mathrm{DM} e_{k}$ and that given by $\mathrm{DM} e_{l}$ on attribute $u_{j}$.

For the attribute $u_{j}$, the average gray relational grade between $\mathrm{DM} e_{k}$ and all other DMs $e_{l}(l \in D, l \neq k)$ is computed as

$$
\eta_{j}^{k}=\frac{1}{t-1} \sum_{l=1, l \neq k}^{t} \gamma\left(\vec{r}_{j}, \vec{r}_{j}^{k}\right)
$$

Thus, the larger the $\eta_{j}^{k}$ is, the more similar the information given by the expert $e_{k}$ is to that implied by the group. Therefore, the bigger the $\lambda_{j}^{k}$. Accordingly, the weight of expert $e_{k}$ with respect to attribute $u_{j}$, denoted by $\lambda_{j}^{k}$, can be defined as

$$
\lambda_{j}^{k}=\frac{\eta_{j}^{k}}{\sum_{l=1}^{t} \eta_{j}^{l}} .
$$

3.2. Integrate Individual Decision Matrices into a Collective Matrix. After the weights of experts are obtained, individual decision matrix $\mathbf{R}_{k}=\left(\widetilde{r}_{k i j}\right)_{m \times n}$ can be integrated into a collective matrix $\widetilde{\mathbf{R}}=\left(\widetilde{r}_{i j}\right)_{m \times n}$ with IVIFWA operator, where

$$
\begin{aligned}
\widetilde{r}_{i j} & =\sum_{k=1}^{t} \widetilde{r}_{i j}^{k} \lambda_{j}^{k} \\
& =\left(\left[1-\prod_{k=1}^{t}\left(1-a_{i j}^{k}\right)^{\lambda_{j}^{k}}, 1-\prod_{k=1}^{t}\left(1-b_{i j}^{k}\right)^{\lambda_{j}^{k}}\right],\right. \\
& {\left.\left[\prod_{k=1}^{t}\left(c_{i j}^{k}\right)^{\lambda_{j}^{k}}, \prod_{k=1}^{t}\left(d_{i j}^{k}\right)^{\lambda_{j}^{k}}\right]\right) . }
\end{aligned}
$$

For convenience, we denote $\widetilde{r}_{i j}$ by

$$
\widetilde{r}_{i j}=\left(\left[a_{i j}, b_{i j}\right],\left[c_{i j}, d_{i j}\right]\right) .
$$

By employing (5), (7), and (8), the score matrix, accuracy matrix, and uncertainty matrix of matrix $\mathbf{R}$ are, respectively, obtained as follows:

$$
\begin{aligned}
\mathbf{S}^{*} & =\left(s_{i j}^{*}\right)_{m \times n}, \\
\mathbf{H} & =\left(h_{i j}\right)_{m \times n}, \\
\boldsymbol{\gamma} & =\left(\gamma_{i j}\right)_{m \times n},
\end{aligned}
$$

where $s_{i j}^{*}=s^{*}\left(\widetilde{r}_{i j}\right), h_{i j}=h\left(\widetilde{r}_{i j}\right)$, and $\gamma_{i j}=\gamma\left(\widetilde{r}_{i j}\right)$.

Utilizing the weighted summation method, we can derive the overall score function, accuracy function, and uncertainty function of alternative $A_{i}$ as

$$
\begin{aligned}
& s_{i}^{*}=\sum_{j=1}^{n} \omega_{j} s_{i j}^{*}, \\
& h_{i}=\sum_{j=1}^{n} \omega_{j} h_{i j}, \\
& \gamma_{i}=\sum_{j=1}^{n} \omega_{j} \gamma_{i j} .
\end{aligned}
$$

If the attribute weights are known in advance, then alternatives can be ranked and selected according to Definition 7. In what follows, a new multiobjective linear programming model is constructed to determine the attribute weights.

3.3. Identify the Attribute Weights by a New Multiobjective Linear Programming Model. Due to the uncertainty of decision making environment and the limited knowledge possessed by experts, experts only may supply partial information about attribute weights. Namely, the information of the attribute weights is incomplete. Let $D$ be the set of incomplete information on attribute weights.

According to Definition 7, the bigger the overall score function (i.e., profit function) $s_{i}^{*}$ and the smaller the overall 
uncertainty function (i.e., risk function) $\gamma_{i}$ of the alternative $A_{i}$, the better the alternative $A_{i}$. Therefore, by maximizing the overall score functions and minimizing the overall uncertainty functions, a multiobjective programming is built to objectively determine the weights of attributes:

$$
\begin{aligned}
\max & \left\{s_{1}^{*}, s_{2}^{*}, \ldots, s_{m}^{*}\right\} \\
\min & \left\{\gamma_{1}, \gamma_{2}, \ldots, \gamma_{m}\right\} \\
\text { s.t. } & \boldsymbol{\omega} \in D .
\end{aligned}
$$

By the max-min method for solving multiobjective programming [38], (25) can be converted as

$$
\begin{array}{ll}
\max & \left\{\min _{i} s_{i}^{*}\right\} \\
\min & \left\{\max _{i} \gamma_{i}\right\} \\
\text { s.t. } & \boldsymbol{\omega} \in D .
\end{array}
$$

From the relationship between $\gamma_{i}$ and $h_{i}$ (see (8)), when $\gamma_{i}$ reaches maximum, $h_{i}$ reaches minimum. Accordingly, minimizing the maximum among $\gamma_{i}$ is equivalent to maximizing the minimum among $h_{i}$. Therefore, (26) can be transformed as

$$
\begin{array}{cl}
\max & \left\{\min _{i} s_{i}^{*}\right\} \\
\max & \left\{\min _{i} h_{i}\right\} \\
\text { s.t. } & \boldsymbol{\omega} \in D .
\end{array}
$$

Assume that $y=\min _{i} s_{i}^{*}, x=\min _{i} h_{i}$; we have $s_{i}^{*} \geq y$ and $h_{i} \geq x$. Thus, by employing (22)-(23), (27) can be rewritten as

$$
\begin{aligned}
\max & y \\
\max & x \\
\text { s.t. } & \sum_{j=1}^{n} s_{i j}^{*} w_{j} \geq y \quad i=1,2, \ldots, m \\
& \sum_{j=1}^{n} h_{i j} w_{j} \geq x \quad i=1,2, \ldots, m \\
& \boldsymbol{\omega} \in D .
\end{aligned}
$$

By the linear weighted summation method, (28) can be converted into the following single objective programming model:

$$
\begin{array}{ll}
\max & \{p y+(1-p) x\} \\
\text { s.t. } & \sum_{j=1}^{n} s_{i j}^{*} w_{j} \geq y \quad i=1,2, \ldots, m \\
& \sum_{j=1}^{n} h_{i j} w_{j} \geq x \quad i=1,2, \ldots, m \\
& \boldsymbol{\omega} \in D,
\end{array}
$$

where $p \in[0,1]$ represents the relative importance of the two objects.

If $0 \leq p<0.5$, then experts are pessimistic and are more concerned about uncertainty function (i.e., risk) than score function (i.e., profit); if $0.5<p \leq 1$, then experts are optimistic and are more concerned about profit than risk; if $p=0.5$, then experts considered that profit is as important as risk.

By solving (29), the vector of attribute weights $\boldsymbol{\omega}=$ $\left(\omega_{1}, \omega_{2}, \ldots, \omega_{m}\right)^{T}$ can be obtained.

\subsection{Decision Process and Algorithm for MAGDM Problems} with IVIFSs. Based on the above analysis, the algorithm and decision process for MAGDM problems are summarized as follows.

Step 1. The experts establish the individual decision matrix $\mathbf{R}^{k}=\left(\widetilde{r}_{i j}^{k}\right)_{m \times n}$ with IVIFSs and supply the set of information on the attribute weights $D$.

Step 2. Calculate the weight of expert $e_{k}$ by (13)-(18), where $k=1,2, \ldots, t ; j=1,2, \ldots, n$.

Step 3. Integrate all individual decision matrix $\mathbf{R}^{k}$ into a collective matrix $\mathbf{R}=\left(\widetilde{r}_{i j}\right)_{m \times n}$ by (19).

Step 4. Derive the score matrix $\mathbf{S}^{*}$, accuracy matrix $\mathbf{H}$, and uncertainty matrix $\boldsymbol{\gamma}$ of the matrix $\mathbf{R}$ by (20)-(21).

Step 5. Determine the weight vector of attributes $\boldsymbol{\omega}$ according to (29).

Step 6. Compute the overall score $s_{i}^{*}$ and uncertainty $\gamma_{i}$ of alternatives $A_{i}$ by (22) and (24).

Step 7. Rank the alternatives and select the best one according to Definition 7.

3.5. The Framework Decision Support System Based on MAGDM with IVIFSs. As the scale of decision making increases, the procedure solving a MAGDM may be complicated. In this case, a decision supporting system (DSS), which is a class of computer-based information system including knowledge-based systems [39, 40], can be formulated to help experts improve their decision-making level and quality through problem analysis, establishment of models, and simulation of decision-making process in a humancomputer interaction way. Figure 1 depicts a framework of DSS designed in this paper for MAGDM with IVIFSs.

As shown in Figure 1, the DSS consists of three modules: User interface, Knowledge base and Model base. Generally, the user interface establishes an interaction between experts and inputs the basic decision information, such as attributes, alternatives and assessment values of alternatives on attributes. The main function of Knowledge base is to help experts perform information transformation and store the corresponding information. For example, the ratings of alternatives on attributes given by experts are transformed 


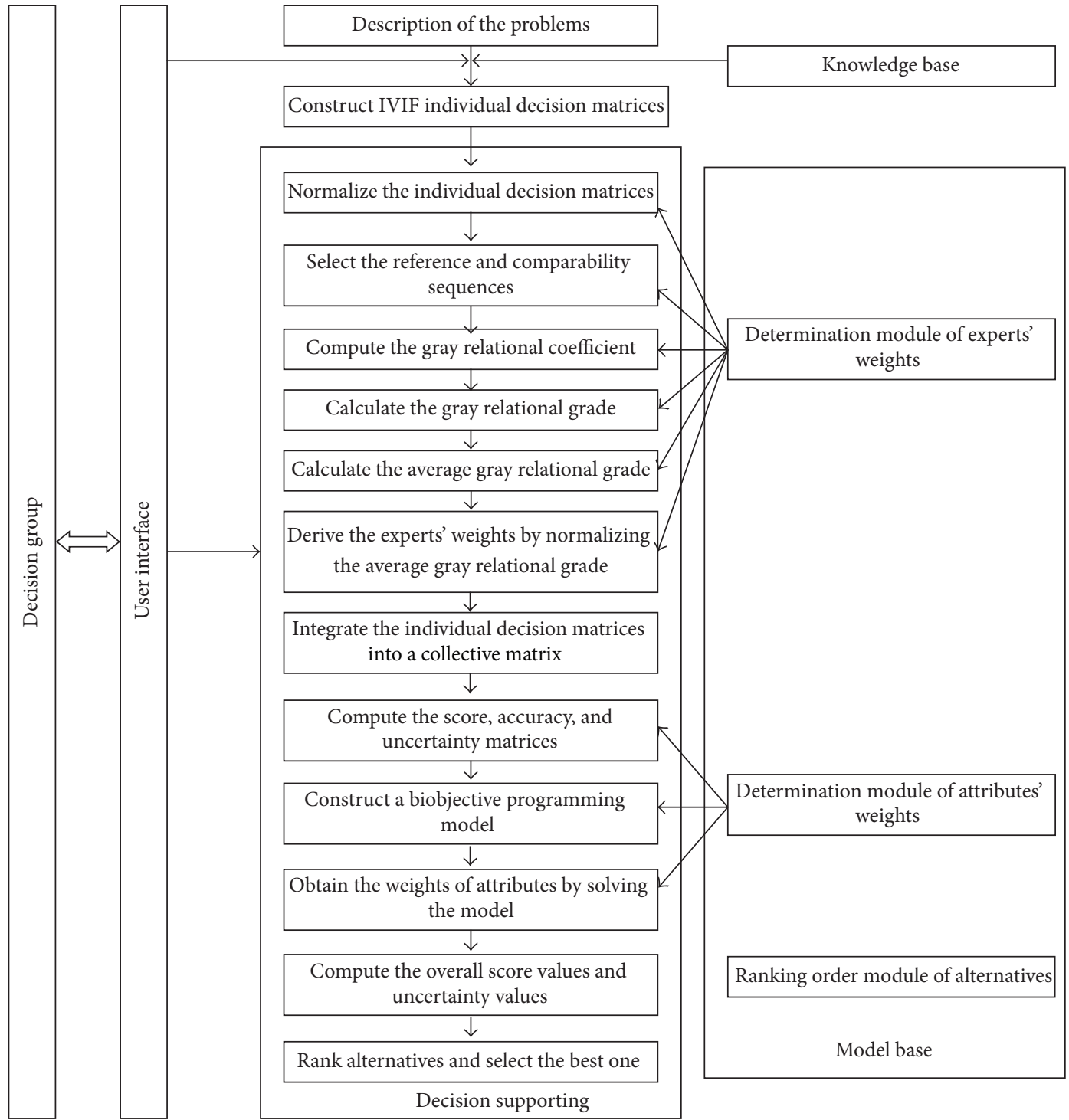

FIGURE 1: Framework of interval-valued intuitionistic fuzzy MAGDM decision supporting system.

into IVIF forms from which individual decision matrices with IVIFSs are constructed and used for the next calculation procedure. Model base involves the methods, such as extended GRA and objective programming as mentioned above. Thus, the ranking of alternatives can be deduced and the optimal decision can be derived by DSS.

\section{A Cloud Service Selection Problem and Comparison Analysis}

In this section, a real cloud service selection problem is given to illustrate the application of the proposed method. Meanwhile, the comparison analysis is also conducted to show the superiority of the proposed method.

\subsection{A Cloud Service Provider Selection Problem and the} Solution Process. Due to the limited technology and capital, an enterprise itself may be unable to build the cloud platform and tries to seek a cloud service to realize its CRM. After the market research and preliminary screening, there are four potential cloud services for further evaluation, including SAP Sales on Demand $\left(A_{1}\right)$, Salesforce Sales Cloud $\left(A_{2}\right)$, Microsoft Dynamic CRM $\left(A_{3}\right)$ and Oracle Cloud CRM $\left(A_{4}\right)$. Four experts $\left(e_{1}, e_{2}, e_{3}, e_{4}\right)$ are invited to evaluate these cloud services on five indicators (attributes), including performance $\left(u_{1}\right)$, payment $\left(u_{2}\right)$, reputation $\left(u_{3}\right)$, scalability $\left(u_{4}\right)$, and security $\left(u_{5}\right)$. In terms of each attribute, each expert has presented his (her) normalized evaluation information for four cloud services in Tables 1-4.

The preference relation set of attributes information supplied by experts is as follows:

$$
\begin{aligned}
D & =\left\{\omega_{1} \leq 2 \omega_{2} ; 0.05 \leq \omega_{2}-\omega_{4} \leq 0.1 ; \omega_{5} \geq 2 \omega_{3} ; \omega_{1}\right. \\
& \left.\leq 0.4 ; \omega_{1}+\omega_{2}+\omega_{3} \geq 0.3 ; \sum_{j=1}^{n} \omega_{j}=1 ; \omega_{j} \geq 0\right\} .
\end{aligned}
$$


TABLE 1: IVIF decision matrix $\mathbf{R}_{1}$.

\begin{tabular}{cccccc}
\hline & $u_{1}$ & $u_{2}$ & $u_{3}$ & $u_{4}$ & $u_{5}$ \\
\hline \multirow{2}{*}{$A_{1}$} & $([0.55,0.65]$, & $([0.35,0.55]$, & $([0.65,0.75]$, & $([0.55,0.75]$, & $([0.10,0.40]$, \\
& $[0.15,0.25])$ & $[0.35,0.45])$ & $[0.15,0.25])$ & $[0.05,0.15])$ & $[0.30,0.50])$ \\
\hline \multirow{2}{*}{$A_{2}$} & $([0.35,0.45]$, & $([0.15,0.35]$, & $([0.35,0.45]$, & $([0.25,0.45]$, & $([0.70,0.80]$, \\
& $[0.25,0.35])$ & $[0.15,0.35])$ & $[0.45,0.55])$ & $[0.45,0.55])$ & $(0.10,0.20])$ \\
\hline \multirow{2}{*}{$A_{3}$} & $([0.55,0.65]$, & $([0.75,0.85]$, & $([0.55,0.85]$, & $([0.45,0.65]$, & $(0.50,0.60]$, \\
& $[0.15,0.25])$ & $[0.05,0.15])$ & $[0.15,0.15])$ & $[0.25,0.35])$ & $(0.20,0.30])$ \\
\hline \multirow{2}{*}{$A_{4}$} & $([0.35,0.55]$, & $([0.15,0.25]$, & $([0.15,0.25]$, & $(0.35,0.45]$, & $(0.20,0.30]$, \\
& $[0.35,0.45])$ & $[0.65,0.75])$ & $[0.55,0.75])$ & $[0.35,0.55])$ & $[0.50,0.60])$ \\
\hline
\end{tabular}

TABLE 2: IVIF decision matrix $\mathbf{R}_{2}$.

\begin{tabular}{cccccc} 
& $u_{1}$ & $u_{2}$ & $u_{3}$ & $u_{4}$ & $u_{5}$ \\
\hline \multirow{2}{*}{$A_{1}$} & $([0.45,0.55]$, & $([0.30,0.40]$, & $([0.55,0.65]$, & $([0.55,0.65]$, & $([0.15,0.35]$, \\
& $[0.25,0.45])$ & $[0.40,0.60])$ & $[0.10,0.15])$ & $[0.05,0.25])$ & $[0.25,0.45])$ \\
\hline \multirow{2}{*}{$A_{2}$} & $([0.35,0.55]$, & $([0.10,0.30]$, & $([0.25,0.35]$, & $([0.25,0.35]$, & $([0.65,0.85]$, \\
& $[0.30,0.40])$ & $[0.30,0.70])$ & $[0.35,0.45])$ & $[0.55,0.65])$ & $(0.05,0.15])$ \\
\hline \multirow{2}{*}{$A_{3}$} & $([0.45,0.65]$, & $([0.60,0.80]$, & $([0.65,0.75]$, & $([0.45,0.65]$, & $([0.55,0.65]$, \\
& $[0.25,0.35])$ & $[0.10,0.20])$ & $[0.05,0.15])$ & $[0.25,0.35])$ & $(0.15,0.35])$ \\
\hline \multirow{2}{*}{$A_{4}$} & $([0.35,0.45]$, & $([0.10,0.20]$, & $([0.05,0.15]$, & $([0.35,0.45]$, & $(0.25,0.45]$, \\
& $[0.35,0.55])$ & $[0.60,0.80])$ & $[0.65,0.75])$ & $[0.35,0.55])$ & $[0.45,0.55])$ \\
\hline
\end{tabular}

Step 1. See Tables 1-4.

Step 2. Calculate the weights of experts.

We take the weights of experts on $u_{1}$ as an example, that is, $\lambda_{1}^{k}(k=1,2,3,4)$, to illustrate the calculating process of the experts' weights. The calculating processes for $\lambda_{1}^{k}$ are as follows:

(i) Select the reference sequence and comparability sequences.

Selecting $\widetilde{\mathbf{r}}_{1}^{1}$ as a reference sequence and $\widetilde{\mathbf{r}}_{1}^{2}, \widetilde{\mathbf{r}}_{1}^{3}, \widetilde{\mathbf{r}}_{1}^{4}$ as comparability sequences, where

$$
\begin{aligned}
\tilde{\mathbf{r}}_{1}^{1}= & \left(\tilde{r}_{11}^{1}, \widetilde{r}_{21}^{1}, \widetilde{r}_{31}^{1}, \widetilde{r}_{41}^{1}\right)=(([0.55,0.65],[0.15,0.25]) \\
& ([0.35,0.45],[0.25,0.35]) \\
& ([0.55,0.65],[0.15,0.25]) \\
& ([0.35,0.55],[0.35,0.45])) \\
\widetilde{\mathbf{r}}_{1}^{2}= & \left(\widetilde{r}_{11}^{2}, \widetilde{r}_{21}^{2}, \widetilde{r}_{31}^{2}, \widetilde{r}_{41}^{2}\right)=(([0.45,0.55],[0.25,0.45]) \\
& ([0.35,0.55],[0.30,0.40]) \\
& ([0.45,0.65],[0.25,0.35]) \\
& ([0.35,0.45],[0.35,0.55])) ; \\
\widetilde{\mathbf{r}}_{1}^{3}= & \left(\widetilde{r}_{11}^{3}, \widetilde{r}_{21}^{3}, \widetilde{r}_{31}^{3}, \widetilde{r}_{41}^{3}\right)=(([0.45,0.75],[0.15,0.25]) \\
& ([0.45,0.55],[0.25,0.45]) \\
& ([0.25,0.45],[0.35,0.45]) \\
& ([0.35,0.45],[0.25,0.45])) ;
\end{aligned}
$$

$$
\begin{aligned}
\widetilde{\mathbf{r}}_{1}^{4}= & \left(\widetilde{r}_{11}^{4}, \widetilde{r}_{21}^{4}, \widetilde{r}_{31}^{4}, \widetilde{r}_{41}^{4}\right)=(([0.65,0.75],[0.15,0.25]), \\
& ([0.40,0.50],[0.40,0.50]) \\
& ([0.40,0.50],[0.30,0.40]) \\
& ([0.30,0.40],[0.40,0.50])) .
\end{aligned}
$$

(ii) Compute the gray relational coefficient matrix.

By (14)-(15), the gray relational coefficient matrix is derived as

$$
\xi_{1}^{1}=\left(\begin{array}{cccc}
0.7122 & 1.000 & 0.8739 & 0.9489 \\
0.9489 & 0.8739 & 0.5115 & 0.9489 \\
0.9489 & 0.7766 & 0.6645 & 0.8739
\end{array}\right)
$$

(iii) Calculate the gray relational grades.

According to (16), we have

$$
\begin{aligned}
& \eta\left(\widetilde{\mathbf{r}}_{1}^{2}, \widetilde{\mathbf{r}}_{1}^{1}\right)=\frac{1}{4} \sum_{i=1}^{4} \xi_{i 1}^{21}=0.8837, \\
& \eta\left(\widetilde{\mathbf{r}}_{1}^{3}, \widetilde{\mathbf{r}}_{1}^{1}\right)=\frac{1}{4} \sum_{i=1}^{4} \xi_{i 1}^{31}=0.8208, \\
& \eta\left(\widetilde{\mathbf{r}}_{1}^{4}, \widetilde{\mathbf{r}}_{1}^{1}\right)=\frac{1}{4} \sum_{i=1}^{4} \xi_{i 1}^{41}=0.8160 .
\end{aligned}
$$

(iv) Determine the average relational grade. 
TABLE 3: IVIF decision matrix $\mathbf{R}_{3}$.

\begin{tabular}{cccccc}
\hline & $u_{1}$ & $u_{2}$ & $u_{3}$ & $u_{4}$ & $u_{5}$ \\
\hline \multirow{2}{*}{$A_{1}$} & $([0.45,0.75]$, & $([0.35,0.55]$, & $([0.60,0.70]$, & $([0.55,0.65]$, & $([0.35,0.55]$, \\
& $[0.15,0.25])$ & $[0.25,0.35])$ & $[0.10,0.20])$ & $[0.05,0.25])$ & $[0.25,0.45])$ \\
\hline \multirow{2}{*}{$A_{2}$} & $([0.45,0.55]$, & $([0.25,0.45]$, & $([0.40,0.50]$, & $([0.15,0.25]$, & $([0.65,0.75]$, \\
& $[0.25,0.45])$ & $[0.35,0.45])$ & $[0.30,0.40])$ & $[0.65,0.75])$ & $[0.15,0.25])$ \\
\hline \multirow{2}{*}{$A_{3}$} & $([0.25,0.45]$, & $([0.65,0.85]$, & $([0.50,0.70]$, & $([0.55,0.75]$, & $([0.65,0.85]$, \\
& $[0.35,0.45])$ & $[0.05,0.15])$ & $[0.10,0.30])$ & $[0.15,0.25])$ & $[0.05,0.15])$ \\
\hline \multirow{2}{*}{$A_{4}$} & $([0.35,0.45]$, & $([0.15,0.25]$, & $([0.10,0.30]$, & $([0.25,0.35]$, & $([0.15,0.25]$, \\
& $[0.25,0.45])$ & $[0.55,0.75])$ & $[0.50,0.70])$ & $[0.45,0.65])$ & $[0.55,0.75])$ \\
\hline
\end{tabular}

TABLE 4: IVIF decision matrix $\mathbf{R}_{4}$.

\begin{tabular}{cccccc}
\hline & $u_{1}$ & $u_{2}$ & $u_{3}$ & $u_{4}$ & $u_{5}$ \\
\hline \multirow{2}{*}{$A_{1}$} & $([0.65,0.75]$, & $([0.30,0.40]$, & $([0.75,0.85]$, & $([0.50,0.60]$, & $([0.15,0.25]$, \\
& $[0.15,0.25])$ & $[0.30,0.40])$ & $[0.05,0.15])$ & $[0.10,0.30])$ & $[0.45,0.65])$ \\
\hline \multirow{2}{*}{$A_{2}$} & $([0.40,0.50]$, & $([0.10,0.20]$, & $([0.35,0.45]$, & $([0.20,0.30]$, & $([0.65,0.75]$, \\
& $[0.40,0.50])$ & $[0.20,0.30])$ & $[0.45,0.55])$ & $[0.40,0.60])$ & $[0.05,0.15])$ \\
\hline \multirow{2}{*}{$A_{3}$} & $([0.40,0.50]$, & $([0.60,0.70]$, & $([0.55,0.85]$, & $([0.40,0.50]$, & $([0.55,0.65]$, \\
& $[0.30,0.40])$ & $[0.10,0.30])$ & $[0.15,0.15])$ & $[0.20,0.30])$ & $[0.25,0.35])$ \\
\hline \multirow{2}{*}{$A_{4}$} & $([0.30,0.40]$, & $([0.10,0.30]$, & $([0.15,0.25]$, & $([0.20,0.30]$, & $([0.35,0.45]$, \\
& $[0.40,0.50])$ & $[0.60,0.70])$ & $[0.55,0.75])$ & $[0.40,0.50])$ & $[0.45,0.55])$ \\
\hline
\end{tabular}

By using (17), the average relational grade between expert $e_{1}$ and all other three experts is obtained as

$$
\eta_{1}^{1}=\frac{1}{3} \sum_{l=2}^{t} \eta\left(\widetilde{r}_{1}^{l}, \widetilde{r}_{1}^{1}\right)=0.8402
$$

Similarly, we can get

$$
\begin{aligned}
& \eta_{1}^{2}=0.7754 \\
& \eta_{1}^{3}=0.8319 \\
& \eta_{1}^{4}=0.7664 .
\end{aligned}
$$

By employing (18), the weights of four experts with respect to $u_{1}$ are derived as:

$$
\begin{aligned}
& \lambda_{1}^{1}=0.2614 \\
& \lambda_{1}^{2}=0.2413 \\
& \lambda_{1}^{3}=0.2588 \\
& \lambda_{1}^{4}=0.2385
\end{aligned}
$$

The calculating processes for the weights of experts with respect to other attributes are omitted, and the results are shown in Table 5.

Step 3. Integrate individual decision matrices $\mathbf{R}_{k}(k=$ $1,2,3,4)$ into a collective decision matrix $\mathbf{R}=\left(\widetilde{r}_{i j}\right)_{m \times n}$ by $(19)$, that is,

$\mathbf{R}$

$$
=\left(\begin{array}{ccccc}
([0.531,0.685],[0.170,0.288]) & ([0.326,0.482],[0.320,0.440]) & ([0.639,0.743],[0.093,0.182]) & ([0.535,0.663],[0.062,0.234]) & ([0.176,0.381],[0.308,0.511]) \\
([0.389,0.514],[0.292,0.420]) & ([0.153,0.332],[0.236,0.425]) & ([0.339,0.439],[0.375,0.476]) & ([0.214,0.341],[0.482,0.628]) & ([0.665,0.796],[0.074,0.178]) \\
([0.423,0.572],[0.249,0.353]) & ([0.658,0.809],[0.070,0.191]) & ([0.568,0.790],[0.099,0.182]) & ([0.459,0.636],[0.209,0.310]) & ([0.556,0.687],[0.154,0.289]) \\
([0.338,0.467],[0.331,0.484]) & ([0.126,0.251],[0.599,0.749]) & ([0.109,0.239],[0.561,0.736]) & ([0.284,0.385],[0.385,0.553]) & ([0.249,0.378],[0.480,0.595])
\end{array}\right)
$$

Step 4. Derive the score matrix, accuracy matrix and uncertainty matrix.

By (5), (7) and (8), the score matrix, accuracy matrix and uncertainty matrix of the collective matrix $\mathbf{R}$ are computed as:

$$
\mathbf{S}^{*}=\left(\begin{array}{ccccc}
0.6898 & 0.5122 & 0.7766 & 0.7254 & 0.4343 \\
0.5477 & 0.4562 & 0.4820 & 0.3586 & 0.8023 \\
0.5980 & 0.8015 & 0.7691 & 0.6440 & 0.7001 \\
0.4975 & 0.2572 & 0.2629 & 0.4326 & 0.3879
\end{array}\right),
$$


TABLE 5: The weights of each expert with respect to different attributes.

\begin{tabular}{cccccc}
\hline & $u_{1}$ & $u_{2}$ & $u_{3}$ & $u_{4}$ & $u_{5}$ \\
\hline$e_{1}$ & 0.2614 & 0.2580 & 0.2176 & 0.2392 & 0.2818 \\
$e_{2}$ & 0.2413 & 0.2422 & 0.2758 & 0.2297 & 0.2757 \\
$e_{3}$ & 0.2588 & 0.2536 & 0.2814 & 0.2162 & 0.1752 \\
$e_{4}$ & 0.2385 & 0.2462 & 0.2252 & 0.3149 & 0.2673 \\
\hline
\end{tabular}

$$
\mathbf{H}=\left(\begin{array}{lllll}
0.8373 & 0.7837 & 0.8284 & 0.7473 & 0.6881 \\
0.8076 & 0.5733 & 0.8143 & 0.8370 & 0.8563 \\
0.7983 & 0.8638 & 0.8194 & 0.8066 & 0.8429 \\
0.8106 & 0.8625 & 0.8221 & 0.8039 & 0.8512
\end{array}\right)
$$

$$
\gamma=\left(\begin{array}{lllll}
0.1625 & 0.2163 & 0.1716 & 0.2527 & 0.3119 \\
0.1924 & 0.4267 & 0.1857 & 0.1630 & 0.1437 \\
0.2017 & 0.1362 & 0.1806 & 0.1934 & 0.1571 \\
0.1894 & 0.1375 & 0.1779 & 0.1961 & 0.1488
\end{array}\right)
$$

Step 5. Determine the attribute weights.

By (28), the following linear programming model is constructed:

$$
\max \{p y+(1-p) x\}\left\{\begin{array}{l}
0.6898 w_{1}+0.5122 w_{2}+0.7766 w_{3}+0.7254 w_{4}+0.4343 w_{5} \geq y \\
0.5477 w_{1}+0.4562 w_{2}+0.4820 w_{3}+0.3586 w_{4}+0.8023 w_{5} \geq y \\
0.5980 w_{1}+0.8015 w_{2}+0.7691 w_{3}+0.6440 w_{4}+0.7001 w_{5} \geq y \\
0.4975 w_{1}+0.2572 w_{2}+0.2629 w_{3}+0.4326 w_{4}+0.3879 w_{5} \geq y \\
0.8373 w_{1}+0.7837 w_{2}+0.8284 w_{3}+0.7473 w_{4}+0.6881 w_{5} \geq x \\
0.8076 w_{1}+0.5733 w_{2}+0.8143 w_{3}+0.8370 w_{4}+0.8563 w_{5} \geq x \\
0.7983 w_{1}+0.8638 w_{2}+0.8194 w_{3}+0.8066 w_{4}+0.8429 w_{5} \geq x \\
0.8106 w_{1}+0.8625 w_{2}+0.8221 w_{3}+0.8039 w_{4}+0.8512 w_{5} \geq x \\
w_{1} \leq 2 w_{2} ; 0.05 \leq w_{2}-w_{4} \leq 0.1 ; w_{5} \geq 2 w_{3} ; w_{1} \leq 0.4 \\
w_{1}+w_{2}+w_{3} \geq 0.3 ; w_{3} \geq 0.05 \\
w_{1}+w_{2}+w_{3}+w_{4}+w_{5}=1 ; \\
w_{1}, w_{2}, w_{3}, w_{4}, w_{5} \geq 0 .
\end{array}\right.
$$

Set $p=0.5$ and solve (39) with Simplex Method. The main components for (39) are as follows:

$$
\begin{aligned}
y & =0.405, \\
x & =0.779, \\
w_{1} & =0.3822, \\
w_{2} & =0.1911, \\
w_{3} & =0.05, \\
w_{4} & =0.1411, \\
w_{5} & =0.2355 .
\end{aligned}
$$

Step 6. Compute the overall score and overall uncertainties of each alternative.

Utilizing (22) and (24), we can calculate the overall scores and uncertainties of all alternatives which are shown in Table 6.
Step 7. Rank alternatives in term of Definition 7. The result of ranking is also listed in Table 6.

From Table 6, it can be seen that alternative $A_{3}$ is the best one, that is, Microsoft Dynamic CRM is the best cloud service.

4.2. Sensitivity Analysis for Parameter $p$. In above example, we get the computation results by a given a priori $(p=$ $0.5)$. However, the attribute weights may vary as the value of weighting coefficient $p$ changes, which may result in different decision results. Hence, it is necessary to do the sensitivity analysis for parameter $p$. The results of sensitivity analysis are depicted in Figure 2.

As shown in Figure 2, when the value of parameter $p$ changes from 0 and 1 , although the overall scores of four providers change slightly, the rankings among the four cloud services remain unchanged. $A_{3}$ is first, followed by $A_{1}$ and followed by $A_{2}$ and the $A_{4}$ is ranked in the last all along. Therefore, we can use (29) freely. 
TABLE 6: The overall scores, accuracies, and ranking of alternatives.

\begin{tabular}{lccc}
\hline Alternative & Score & Uncertainty & Ranking \\
\hline$A_{1}$ & 0.6050 & 0.2213 & 2 \\
$A_{2}$ & 0.5602 & 0.2213 & 3 \\
$A_{3}$ & 0.6759 & 0.1765 & 1 \\
$A_{4}$ & 0.4048 & 0.1704 & 4 \\
\hline
\end{tabular}

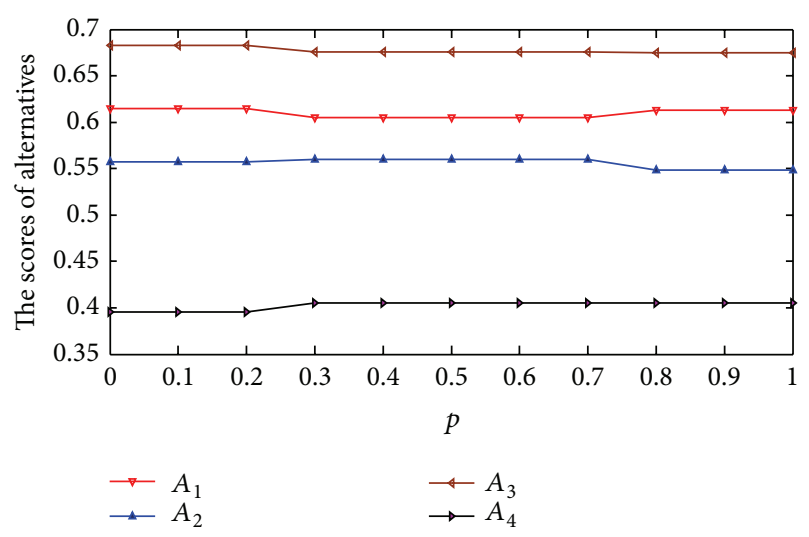

FIGURE 2: The overall scores of four candidate providers with respect to $p$.
4.3. Comparison Analysis with the Method Using the Score Function Given by $\mathrm{Xu}$ [30]. In the above cloud service selection example, if the scores of alternatives are computed with the score function given by $\mathrm{Xu}$ [30] (see (4)), then the score matrix is given as
S

$$
=\left(\begin{array}{ccccc}
0.3795 & 0.0244 & 0.5532 & 0.4508 & -0.1313 \\
0.0954 & -0.0876 & -0.0361 & -0.2828 & 0.6047 \\
0.1959 & 0.6029 & 0.5382 & 0.2880 & 0.4002 \\
-0.0050 & -0.4856 & -0.4742 & -0.1349 & -0.2241
\end{array}\right) .
$$

The accuracy matrix retains unchanged. Putting the score matrix $\mathbf{S}$ and accuracy matrix $\mathbf{H}$ into (29), we get the following programming model:

$$
\max \{p y+(1-p) x\}\left\{\begin{array}{l}
0.3795 w_{1}+0.0244 w_{2}+0.5532 w_{3}+0.4508 w_{4}-0.1313 w_{5} \geq y \\
0.0954 w_{1}-0.0876 w_{2}-0.0361 w_{3}-0.2828 w_{4}+0.6047 w_{5} \geq y \\
0.1959 w_{1}+0.6029 w_{2}+0.5382 w_{3}+0.2880 w_{4}+0.4002 w_{5} \geq y \\
-0.005 w_{1}-0.4856 w_{2}-0.4742 w_{3}-0.1349 w_{4}-0.2241 w_{5} \geq y \\
0.8373 w_{1}+0.7837 w_{2}+0.8284 w_{3}+0.7473 w_{4}+0.6881 w_{5} \geq x \\
0.8076 w_{1}+0.5733 w_{2}+0.8143 w_{3}+0.8370 w_{4}+0.8563 w_{5} \geq x \\
0.7983 w_{1}+0.8638 w_{2}+0.8194 w_{3}+0.8066 w_{4}+0.8429 w_{5} \geq x \\
0.8106 w_{1}+0.8625 w_{2}+0.8221 w_{3}+0.8039 w_{4}+0.8512 w_{5} \geq x \\
w_{1} \leq 2 w_{2} ; 0.05 \leq w_{2}-w_{4} \leq 0.1 ; w_{5} \geq 2 w_{3} ; w_{1} \leq 0.4 ; \\
w_{1}+w_{2}+w_{3} \geq 0.3 ; w_{3} \geq 0.05 \\
w_{1}+w_{2}+w_{3}+w_{4}+w_{5}=1 ; \\
w_{1}, w_{2}, w_{3}, w_{4}, w_{5} \geq 0
\end{array}\right.
$$

Still let $p=0.5$, by employing the Lingo Soft, we find that (42) has no feasible solution. Thus, the ranking order of alternatives cannot be obtained. This shows that introducing the normalized score function proposed in this paper is very important.

\section{Conclusions}

In order to stand out in the fierce competition, more and more enterprises begin to select cloud service as one of their development strategy. Cloud service selection can be regarded as a kind of MAGDM. In this paper, we have studied the cloud service selection problems with IVIFSs and incomplete information on attribute weights. A novel MAGDM method was proposed to solve this kind of GDM problems. There are following three dramatic features in the proposed method.

(1) The assessment values of alternatives on attributes are in the form of IVIFSs which can help experts express their preferences more flexibly. 
(2) By extending the classical GRA method into IVIF environment, a new approach is presented to determine the weights of experts. Furthermore, the weights of each expert obtained are different on different attributes, which is much closer to the real-world decision situation.

(3) A multiobjective programming model is constructed to derive the weights of attributes.

The future work of this study is to apply the proposed method to other management areas, such as risk investment, material selection and so on.

\section{Conflict of Interests}

The authors declare that there is no conflict of interests regarding the publication of this paper.

\section{Acknowledgments}

The authors would like to thank Associate Professor. Jiu-Ying Dong for improving the linguistic quality of this paper. This research was supported by the National Natural Science Foundation of China (nos. 71061006, 71161011, 61263018, 71361002 and 11461030), the National 863 Plan Project (2013AA12A402), the Humanities Social Science Programming Project of Ministry of Education of China (no. 09YGC630107), the Natural Science Foundation of Jiangxi Province of China (nos. 20114BAB201012 and 20142BAB201011), "Twelve five" Programming Project of Jiangxi province Social Science (2013) (no. 13GL17), the Natural Science Foundation of Guangxi (GXNSF) (2013AA019349 and 2013AA278003), Science Foundation of Guangxi Educational Committee (YB2014150) and the Excellent Young Academic Talent Support Program of Jiangxi University of Finance and Economics.

\section{References}

[1] L. Sun, H. Dong, F. K. Hussain, O. K. Hussain, and E. Chang, "Cloud service selection: state-of-the-art and future research directions," Journal of Network and Computer Applications, vol. 45, pp. 134-150, 2014.

[2] S. Ding, C. Xia, Q. Cai, K. Zhou, and S. Yang, "QoS-aware resource matching and recommendation for cloud computing systems," Applied Mathematics and Computation, vol. 247, no. 15, pp. 941-950, 2014.

[3] A. Goscinski and M. Brock, "Toward dynamic and attribute based publication, discovery and selection for cloud computing," Future Generation Computer Systems, vol. 26, no. 7, pp. 947-970, 2010.

[4] A. Jula, E. Sundararajan, and Z. Othman, "Cloud computing service composition: a systematic literature review," Expert Systems with Applications, vol. 41, no. 8, pp. 3809-3824, 2014.

[5] J. Siegel and J. Perdue, "Cloud services measures for global use: the Service Measurement Index (SMI)," in Proceedings of the Annual SRII Global Conference (SRII '12), pp. 411-415, IEEE, San Jose, Calif, USA, July 2012.

[6] S. K. Garg, S. Versteeg, and R. Buyya, "SMICloud: a framework for comparing and ranking cloud services," in Proceedings of the 4th IEEE/ACM international conference on utility and Cloud on utility and Cloud computing (UCC' 11), pp. 210-218, Melbourne, Australia, December 2011.

[7] M. Menzel, M. Schönherr, and S. Tai, " $\left(\mathrm{MC}^{2}\right)^{2}$ : criteria, requirements and a software prototype for Cloud infrastructure decisions," Software: Practice and Experience, vol. 43, no. 11, pp. 1283-1297, 2013.

[8] N. Limam and R. Boutaba, "Assessing software service quality and trustworthiness at selection time," IEEE Transactions on Software Engineering, vol. 36, no. 4, pp. 559-574, 2010.

[9] S. Silas, E. B. Rajsingh, and K. Ezra, "Efficient Service Selection middleware using ELECTRE methodology for cloud environments," Information Technology Journal, vol. 11, no. 7, pp. 868875, 2012.

[10] P. Saripalli and G. Pingali, "MADMAC: multiple attribute decision methodology for Adoption of clouds," in Proceedings of the IEEE 4th International Conference on Cloud Computing (CLOUD '11), pp. 316-323, Washington, DC, USA, July 2011.

[11] L. Zhao, Y. Ren, M. Li, and K. Sakurai, "Flexible service selection with user-specific QoS support in service-oriented architecture," Journal of Network and Computer Applications, vol. 35, no. 3, pp. 962-973, 2012.

[12] C.-W. Chang, P. Liu, and J.-J. Wu, "Probability-based cloud storage providers selection algorithms with maximum availability," in Proceedings of the 41st International Conference on Parallel Processing (ICPP '12), pp. 199-208, Pittsburgh, Pa, USA, September 2012.

[13] S. Sundareswaran, A. Squicciarini, D. Lin, and S. Sundareswaran, "A brokerage-based approach for cloud service selection," in Proceedings of the IEEE 5th International Conference on Cloud Computing (CLOUD '12), pp. 558-565, Honolulu, Hawaii, USA, June 2012.

[14] Q. He, J. Han, Y. Yang, J. Grundy, and H. Jin, "QoS-driven service selection for multi-tenant SaaS," in Proceedings of the International Conference on Cloud Computing (CLOUD '12), pp. 24-29, Honolulu, Hawaii, USA, June 2012.

[15] B. Martens, F. Teuteberg, and M. Gräuler, "Design and implementation of a community platform for the evaluation and selection of Cloud computing services: a market analysis," in Proceedings of the 19th European Conference on Information Systems (ECIS '11), p. 215, Helsinki, Finland, June 2011.

[16] J. Yang, W. M. Lin, and W. C. Dou, "An adaptive service selection method for cross-cloud service composition," Concurrency and Computation: Practice and Experience, vol. 25, no. 18, pp. 24352454, 2013.

[17] G. L. Xu and F. Liu, "An approach to group decision making based on interval multiplicative and fuzzy preference relations by using projection," Applied Mathematical Modelling, vol. 37, no. 6, pp. 3929-3943, 2013.

[18] S.-P. Wan and D.-F. Li, "Fuzzy LINMAP approach to heterogeneous MADM considering comparisons of alternatives with hesitation degrees," Omega, vol. 41, no. 6, pp. 925-940, 2013.

[19] S.-P. Wan and J.-Y. Dong, "Possibility linear programming with trapezoidal fuzzy numbers," Applied Mathematical Modelling, vol. 38, no. 5-6, pp. 1660-1672, 2014.

[20] H. Akdag, T. Kalaycı, S. Karagöz, H. Zülfikar, and D. Giz, “The evaluation of hospital service quality by fuzzy MCDM," Applied Soft Computing, vol. 23, pp. 239-248, 2014.

[21] L. A. Zadeh, "Fuzzy sets," Information and Computation, vol. 8, no. 3, pp. 338-353, 1965.

[22] K. T. Atanassov, "Intuitionistic fuzzy sets," Fuzzy Sets and Systems, vol. 20, no. 1, pp. 87-96, 1986. 
[23] K. Atanassov and G. Gargov, "Interval valued intuitionistic fuzzy sets," Fuzzy Sets and Systems, vol. 31, no. 3, pp. 343-349, 1989.

[24] S.-P. Wan and J.-Y. Dong, "Power geometric operators of trapezoidal intuitionistic fuzzy numbers and application to multiattribute group decision making," Applied Soft Computing, vol. 29, pp. 153-168, 2015.

[25] S. P. Wan, W. Feng, L. L. Lin, and J. Y. Dong, "An intuitionistic fuzzy linear programming method for logistics outsourcing provider selection," Knowledge-Based Systems, vol. 82, pp. 8094, 2015.

[26] S. P. Wan and J. Y. Dong, "Interval-valued intuitionistic fuzzy mathematical programming method for hybrid multi-criteria group decision making with interval-valued intuitionistic fuzzy truth degrees," Information Fusion, vol. 26, pp. 49-65, 2015.

[27] S. P. Wan, G. L. Xu, F. Wan, and J. Y. Dong, "A new method for Atanassov's interval-valued intuitionistic fuzzy MAGDM with incomplete attribute weight information," Information Sciences, vol. 316, pp. 329-347, 2015.

[28] S.-P. Wan and D.-F. Li, "Atanassov's intuitionistic fuzzy programming method for heterogeneous multiattribute group decision making with atanassov's intuitionistic fuzzy truth degrees," IEEE Transactions on Fuzzy Systems, vol. 22, no. 2, pp. 300-312, 2014.

[29] J. L. Deng, Gray System Theory, Huazhong University of Science and Technology Press, Wuhan, China, 2002.

[30] Z.-S. Xu, "Methods for aggregating interval-valued intuitionistic fuzzy information and their application to decision making," Control and Decision, vol. 22, no. 2, pp. 215-219, 2007 (Chinese).

[31] G.-D. Li, D. Yamaguchi, and M. Nagai, "A grey-based decisionmaking approach to the supplier selection problem," Mathematical and Computer Modelling, vol. 46, no. 3-4, pp. 573-581, 2007.

[32] W. J. Gu, Z. C. Sun, X. Z. Wei, and H. F. Dai, "A new method of accelerated life testing based on the Grey System Theory for a model-based lithium-ion battery life evaluation system," Journal of Power Sources, vol. 267, pp. 366-379, 2014.

[33] H. B. Kuang, D. M. Kilgour, and K. W. Hipel, "Grey-based PROMETHEE II with application to evaluation of source water protection strategies," Information Sciences, vol. 294, no. 10, pp. 376-389, 2015.

[34] F. Y. Meng, Q. Zhang, and X. H. Cheng, "Approaches to multiple-criteria group decision making based on intervalvalued intuitionistic fuzzy Choquet integral with respect to the generalized $\lambda$-Shapley index," Knowledge-Based Systems, vol. 37, pp. 237-249, 2013.

[35] S. Wan and J. Dong, "A possibility degree method for intervalvalued intuitionistic fuzzy multi-attribute group decision making," Journal of Computer and System Sciences, vol. 80, no. 1, pp. 237-256, 2014.

[36] Z. L. Yue and Y. Jia, "An application of soft computing technique in group decision making under interval-valued intuitionistic fuzzy environment," Applied Soft Computing, vol. 13, no. 5, pp. 2490-2503, 2013.

[37] X. L. Zhang and Z. S. Xu, "Soft computing based on maximizing consensus and fuzzy TOPSIS approach to intervalvalued intuitionistic fuzzy group decision making," Applied Soft Computing, vol. 26, pp. 42-56, 2015.

[38] D. F. Li, Multiobjective Many-Person Decision Makings and Games, National Defense Industry Press, Beijing, China, 2003.
[39] S. Cebi and C. Kahraman, "Developing a group decision support system based on fuzzy information axiom," KnowledgeBased Systems, vol. 23, no. 1, pp. 3-16, 2010.

[40] G. Qian, H. Wang, and X. Q. Feng, "Generalized hesitant fuzzy sets and their application in decision support system," Knowledge-Based Systems, vol. 37, pp. 357-365, 2013. 


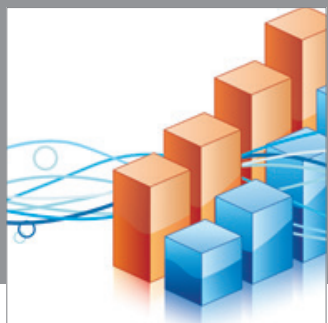

Advances in

Operations Research

mansans

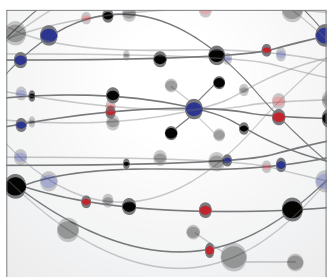

The Scientific World Journal
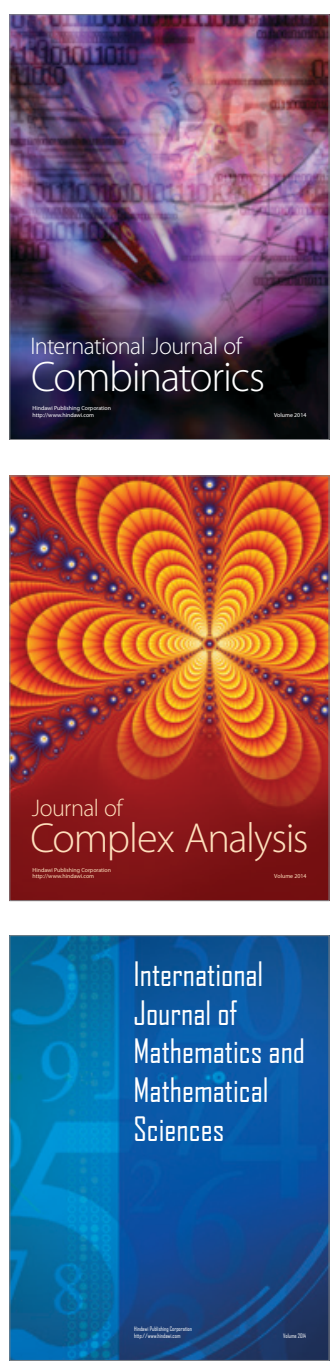
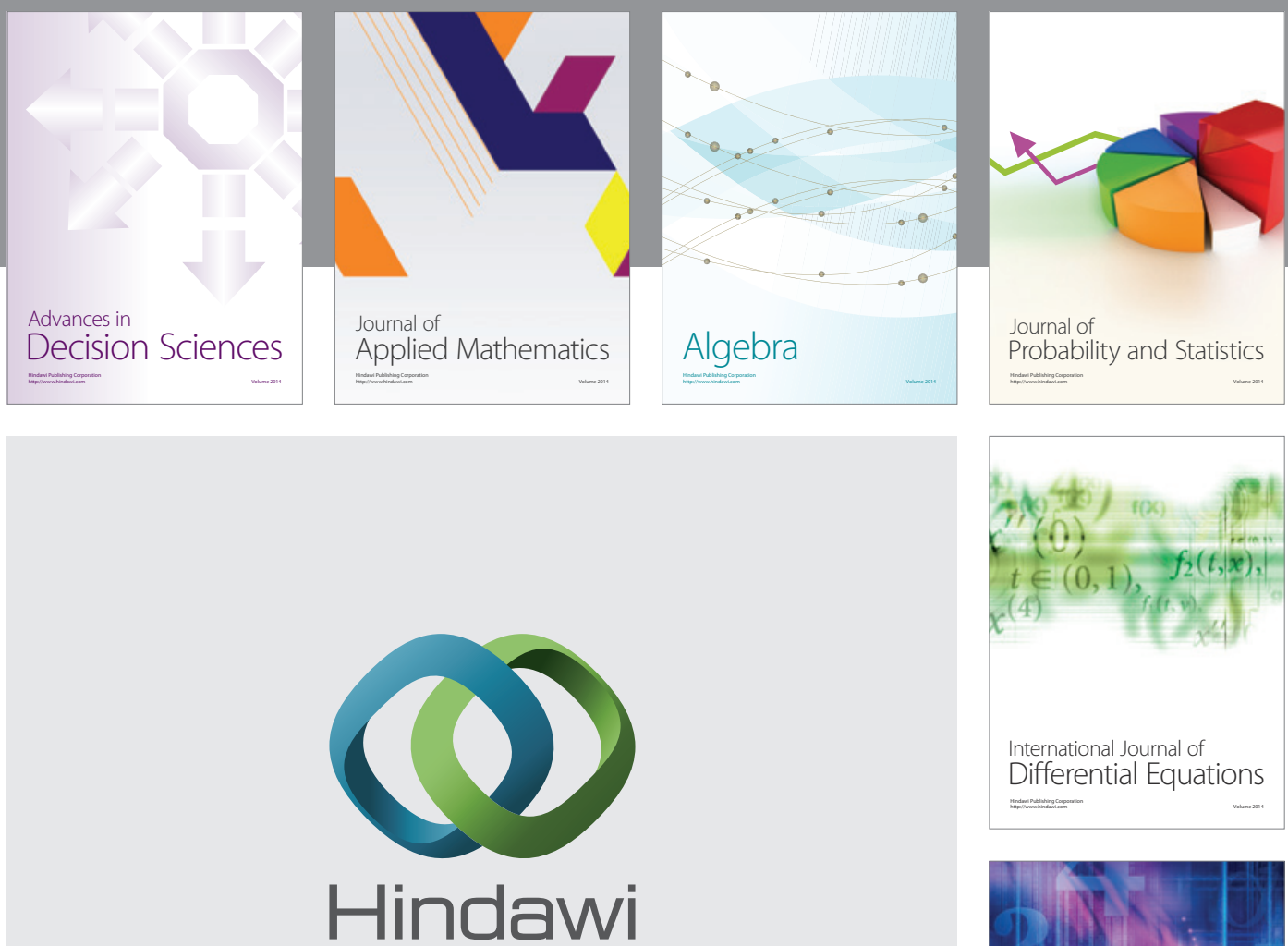

Submit your manuscripts at http://www.hindawi.com
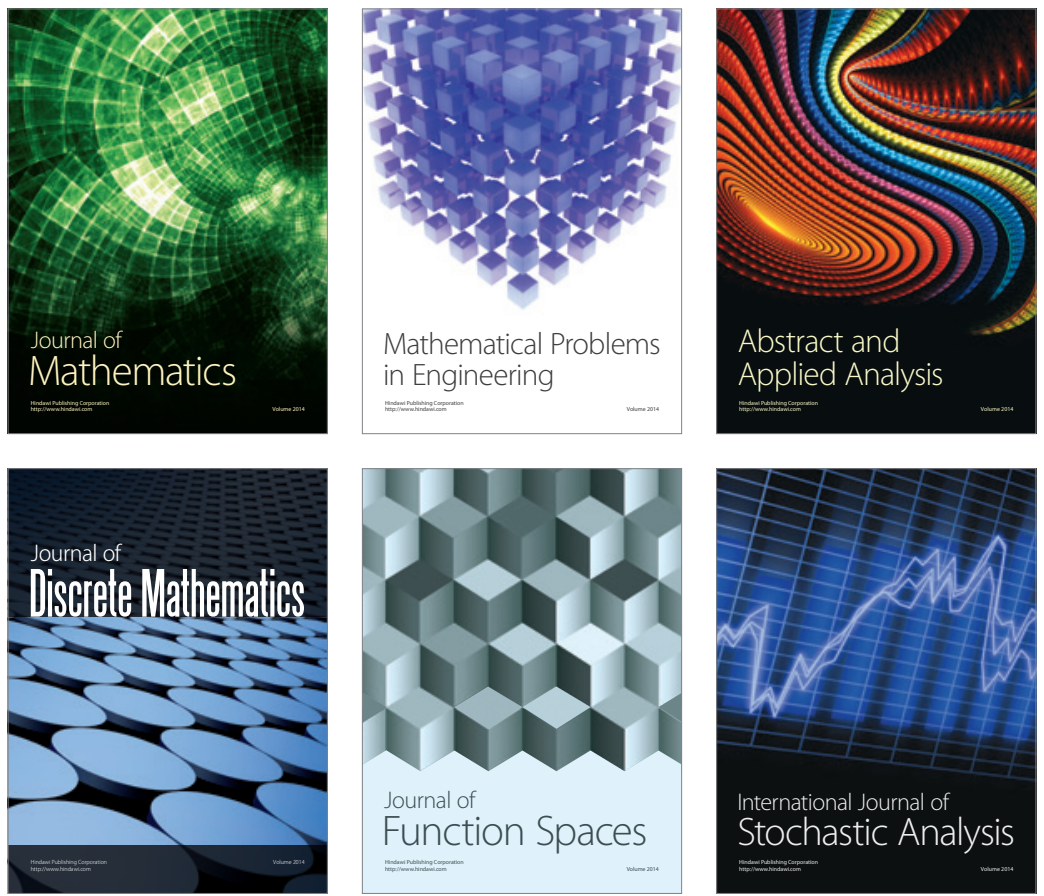

Journal of

Function Spaces

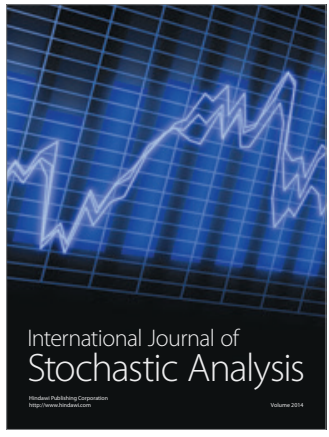

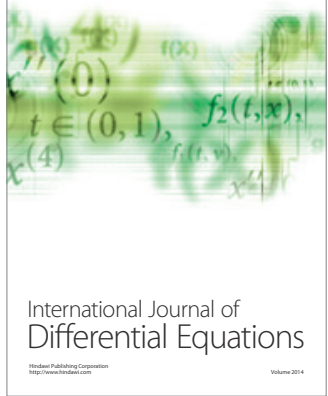
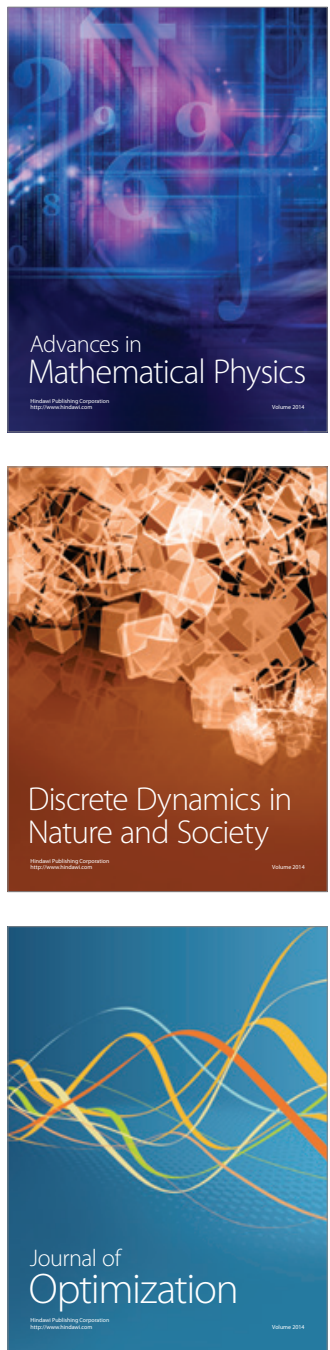